\title{
Transhiatal Esophagectomy for Distal and Cardia Cancers: Implications of a Positive Gastric Margin
}

\author{
Paul D. DiMusto, MD, and Mark B. Orringer, MD \\ Section of Thoracic Surgery, Department of Surgery, University of Michigan Medical Center, Ann Arbor, Michigan
}

Background. A common operation for cancer of the esophagus and cardia consists of transhiatal esophagectomy, proximal gastrectomy, and a cervical esophagogastric anastomosis. The oncologic adequacy of dividing the stomach 4 to $6 \mathrm{~cm}$ distal to palpable tumor is not well documented, and when a positive gastric margin is present on the final pathologic analysis, the appropriate management is not established. This study was undertaken to determine the incidence of a positive gastric margin in these patients and the impact of adjuvant treatment.

Methods. A retrospective review was performed of 1044 patients undergoing transhiatal esophagectomy for adenocarcinoma of the distal esophagus or cardia. Twenty (1.9\%) had a positive gastric margin on final the pathologic evaluation and met inclusion criteria for this study.

Results. Nine patients (45\%) received adjuvant therapy consisting of radiation in 3, chemotherapy in 4, or both in 2 .

$\mathrm{T}^{\mathrm{s}}$ he optimal surgical treatment of adenocarcinoma of the distal esophagus and gastric cardia remains a challenge that is still debated in the literature. Some suggest that these are in fact two different clinical entities that require different surgical approaches. Siewert and colleagues [1] advocate an esophagectomy and proximal partial gastrectomy for tumors originating above the gastroesophageal junction (GEJ) and a distal esophagectomy and extended gastrectomy for tumors originating at the GEJ or distal to it. Others recommend a transhiatal esophagectomy (THE) as the operation of choice for resection of cancer at the GEJ [2].

At the University of Michigan, the preferred operative approach to carcinoma of the distal esophagus and cardia is a THE, proximal partial gastrectomy, and a cervical esophagogastric anastomosis (CEGA) [3]. We define the area of the cardia as being within $2 \mathrm{~cm}$ of the GEJ. In most of these patients, a negative distal gastric surgical margin is achieved. A small percentage, however, are found to have a positive gastric margin of resection on the final pathologic analysis. To date, there is no consensus on the appropriate

\footnotetext{
Accepted for publication Sept 6, 2006.

Presented at the Forty-third Annual Meeting of The Society of Thoracic Surgeons, San Diego, CA, Jan 29-31, 2007.

Address correspondence to Dr Orringer, Section of Thoracic Surgery, University of Michigan Medical Center, 1500 E Medical Center Dr, 2120 Taubman Center, Box 0344, Ann Arbor, MI 48109; e-mail: morrin@ umich.edu.
}

Their average postoperative survival was 477 days, compared with 455 days in those not receiving adjuvant therapy ( $p=0.898)$. Local tumor recurrence developed in 1 patient (11\%) in the treatment group and in $3(27 \%)$ in the no treatment group ( $p=0.386$ ).

Conclusions. A transhiatal esophagectomy and proximal gastrectomy for carcinoma of the distal esophagus and cardia, dividing the stomach 4 to $6 \mathrm{~cm}$ from palpable tumor, provides a negative gastric margin in $98 \%$ of patients. In the few patients who have a positive gastric margin, $80 \%$ die with distant metastases, which would not be influenced by more extensive gastric resection, and in about $20 \%$, local tumor recurrence develops in the intrathoracic stomach, seldom causing dysphagia. Adjuvant therapy for a positive gastric margin neither improves survival nor reduces local tumor recurrence.

(Ann Thorac Surg 2007;83:1993-9) (C) 2007 by The Society of Thoracic Surgeons

management of these patients. Adjuvant chemotherapy, radiation therapy, combined chemoradiation therapy, or no additional treatment have all been used at our institution to treat patients with a positive gastric margin.

Although currently no reports in the literature specifically address this issue, several do address related issues. One examining the effect of adjuvant chemoradiation after esophagectomy in patients with locoregionally advanced cancer of the esophagus showed a significant increase in survival time, an increase in median time to recurrence, and increased recurrence-free survival time compared with propensity-matched controls [4]. This study, however, did not analyze separately those patients who had a $\mathrm{R} 0$ resection compared with those who had a R1 resection.

A prospective randomized study involving 495 patients examined the benefit of postoperative radiation therapy after radical resection for squamous cell carcinoma of the thoracic esophagus [5]. No significant difference was found in survival rates between patients undergoing surgery and radiation therapy compared with surgery alone when all stages of cancer were included; however, when the authors examined only those patients with stage III disease, they did find a significant increase in survival in the patients who received adjuvant radiation in addition to surgery.

Another prospective randomized study that compared adjuvant radiotherapy with adjuvant chemotherapy in patients who had undergone a curative resection for esophageal carcinoma found no significant difference in 5-year survival between the two groups [6]. This study 
Table 1. Positive Distal Gastric Margin of Resection in Patients Undergoing a Transhiatal Esophagectomy for Adenocarcinoma of the Distal Esophagus and Cardia in 20 Patients

\begin{tabular}{lccc}
\hline & $\begin{array}{c}\text { Adjuvant } \\
\text { Therapy } \\
(\mathrm{n}=9)\end{array}$ & $\begin{array}{c}\text { No Adjuvant } \\
\text { Therapy } \\
(\mathrm{n}=11)\end{array}$ & $p$ Value \\
\hline $\begin{array}{l}\text { Age at presentation } \\
\text { (years) }\end{array}$ & $68.1 \pm 12.3$ & $67.6 \pm 8.0$ & 0.903 \\
$\begin{array}{l}\text { Gender } \\
\text { Men }\end{array}$ & 6 & 10 & 0.285 \\
$\quad$ Women & 3 & 1 & \\
$\begin{array}{l}\text { Final pathologic Stage } \\
\text { II }\end{array}$ & 0 & 2 & 0.479 \\
$\quad$ III & 9 & 9 & \\
$\begin{array}{l}\text { Neoadjuvant radiation } \\
\text { therapy }\end{array}$ & 2 & 2 & 1.00 \\
$\begin{array}{l}\text { Neoadjuvant } \\
\text { chemotherapy }\end{array}$ & 2 & 2 & 1.00 \\
\hline
\end{tabular}

included patients who had cancer in all portions of the esophagus and all stages of cancer.

Finally, a report of patients with squamous cell cancer of the esophagus who had a positive proximal margin of resection found no significant difference in anastomotic recurrences compared with patients with a negative proximal margin of resection [7]. These authors also reported that among those with a positive margin, adjuvant radiotherapy did not significantly affect the chance of anastomotic recurrence. They also found that those with a positive margin who received adjuvant radiation therapy had a shorter median survival time compared with those who did not receive adjuvant radiation, although this difference was not significant.

The present study was undertaken to review the clinical course of our patients with a positive distal gastric margin after THE, proximal gastrectomy, and CEGA for adenocarcinoma of the distal one third of the esophagus and gastric cardia and to investigate the potential benefits of adjuvant therapy in preventing local tumor recurrence, improving functional swallowing outcome, and prolonging survival in these patients.

\section{Patients and Methods}

Between 1975 and September 20, 2005, 1986 patients underwent a transhiatal esophagectomy, proximal partial gastrectomy, and cervical esophagogastric anastomosis on the thoracic surgery service at the University of Michigan; 474 (24\%) were for benign disease and 1512 $(76 \%)$ were for malignant disease. Adenocarcinoma of the distal one third of the esophagus or the gastric cardia was found in $1044(69 \%)$ of the patients with carcinoma. A positive distal gastric margin of resection was found on final pathologic analysis of the resected specimens in 26 $(2.5 \%)$ of these latter patients, and they are the focus of this study. The University of Michigan Medical School Institutional Review Board approved this retrospective the study on September 8, 2005 and waived the need for individual informed consent.

Of the 26 patients with a positive gastric margin, 20 met inclusion criteria for the study and had adequate follow-up data available. Four of the 26 patients had stage IV disease and were excluded because they had documented metastatic disease, and residual tumor at the gastric margin should not have impacted their survival. Another patient was excluded because gross tumor was knowingly left behind in the operation, which was done only for palliation of dysphagia. Finally, one patient with gastric tip necrosis underwent an emergent completion gastrectomy to control massive bleeding. He had a staged total gastrectomy rather than a partial gastrectomy and therefore was excluded.

As has been our practice, the stomach was divided 4 to $6 \mathrm{~cm}$ from palpable tumor, and the residual stomach was positioned in the posterior mediastinum in the esophageal bed in all 20 patients. A frozen section is not routinely obtained at our institution, and no frozen sections were done during the operation in the 20 patients in this study.

The 20 patients included in this study have died; therefore, follow-up was completed by using the University of Michigan Esophagectomy Database and the patients' medical records, when available. To ensure adequate follow-up, our patients are routinely contacted by telephone and the information in our database is up-

Table 2. Adjuvant Therapy for a Positive Gastric Margin-Outcomes in 9 Patients

\begin{tabular}{|c|c|c|c|c|c|c|c|c|}
\hline Patient & Radiation & Chemo & $\begin{array}{l}\text { Documented } \\
\text { Local } \\
\text { Recurrence }\end{array}$ & $\begin{array}{l}\text { Survival } \\
\text { Time } \\
\text { (Days) }\end{array}$ & $\begin{array}{l}\text { Metastatic } \\
\text { Disease }\end{array}$ & Cause of Death & $\begin{array}{l}\text { Swallowing } \\
\text { Function }\end{array}$ & $\begin{array}{c}\text { Siewert } \\
\text { Class }\end{array}$ \\
\hline 1 & Yes & No & No & 1350 & Yes & Presumed Metastases & Good & I \\
\hline 2 & Yes & No & No & 191 & Yes & Metastases & Excellent & I \\
\hline 3 & Yes & No & No & 310 & Yes & Presumed Metastases & Excellent & I \\
\hline 4 & No & Yes & No & 716 & Presumed & Presumed Metastases & Excellent & I \\
\hline 5 & No & Yes & No & 233 & Presumed & Presumed Metastases & Fair & I \\
\hline 6 & No & Yes & No & 720 & No & Heart Disease & Poor & I \\
\hline 7 & No & Yes & No & 302 & Yes & Metastases & Excellent & I \\
\hline 8 & Yes & Yes & Yes & 180 & Yes & Metastases & Poor & I \\
\hline 9 & Yes & Yes & No & 288 & Yes & Metastases & Excellent & I \\
\hline
\end{tabular}


Table 3. No Adjuvant Therapy for a Positive Gastric Margin-Outcomes in 11 Patients

\begin{tabular}{|c|c|c|c|c|c|c|}
\hline Patient & $\begin{array}{l}\text { Documented } \\
\text { Local } \\
\text { Recurrence }\end{array}$ & $\begin{array}{l}\text { Survival } \\
\text { Time } \\
\text { (Days) }\end{array}$ & $\begin{array}{l}\text { Metastatic } \\
\text { Disease }\end{array}$ & Cause of Death & $\begin{array}{l}\text { Swallowing } \\
\text { Function }\end{array}$ & $\begin{array}{c}\text { Siewert } \\
\text { Class }\end{array}$ \\
\hline 1 & No & 991 & Yes & Metastases & Excellent & I \\
\hline 2 & No & 145 & Yes & Metastases & Excellent & I \\
\hline 3 & No & 398 & Yes & Metastases & Fair & II \\
\hline 4 & Yes & 380 & Yes & Metastases & Poor & III \\
\hline 5 & No & 111 & Yes & Presumed Metastases & Excellent & I \\
\hline 6 & No & 152 & No & Flu & Excellent & I \\
\hline 7 & No & 195 & Yes & Presumed Metastases & Excellent & II \\
\hline 8 & Yes & 354 & Presumed & Presumed Metastases & Fair & I \\
\hline 9 & No & 289 & Presumed & Presumed Metastases & Poor & I \\
\hline 10 & Yes & 1474 & Presumed & Presumed Metastases & Poor & II \\
\hline 11 & No & 520 & No & Stroke & Unknown & II \\
\hline
\end{tabular}

dated, even though the patients are no longer seen in our outpatient clinic.

The swallowing function of each patient at last follow-up was scored as follows: excellent, no symptoms; good, mild symptoms that did not require treatment; fair, symptoms requiring occasional treatment, such as an esophageal dilatation for periodic dysphagia; and poor, ongoing symptoms requiring regular treatment, such as repeated regular dilatations for dysphagia. The swallowing score for each patient was based on data gathered at the time of last contact; given their relatively short survival time, this was within a few months of death for most of the patients included in the analysis.

Statistical analysis was conducted using SPSS 13.0 (SPSS Inc, Chicago, IL). The 20 patients were divided into two groups for statistical analysis: those who received some form of adjuvant treatment and those who did not. A $t$ test was used to compare the two groups in terms of age at presentation. A $\chi^{2}$ test was used to compare the two groups in terms of gender, pathologic stage of cancer, number receiving neoadjuvant radiation therapy, and number receiving neoadjuvant chemotherapy. A KaplanMeier analysis of survival was used to compare the two groups. A log-rank test was used to compare the KaplanMeier functions between groups. The Fisher exact test was used to look for a difference in the incidence of local tumor recurrence between the groups. A Mann-Whitney test was used to compare swallowing function between the two groups. The results from the statistical analysis of the two groups are the focus of this paper.

\section{Results}

Sixteen men and four women met inclusion criteria and were analyzed. Two $(10 \%)$ of the 20 patients had pathologic stage II disease, and $18(90 \%)$ had stage III disease by the TNM pathologic classification. Nine $(45 \%)$ patients received some form of adjuvant treatment because of their positive gastric margin. The other $11(55 \%)$ received no adjuvant treatment. Four $(20 \%)$ patients received neoadjuvant radiation and chemotherapy under various research protocols. Two of these latter patients also received adjuvant therapy. The average age of the adjuvant therapy group was $68.1 \pm 12.3$ years (range, 39 to 80 years) and that of the group with no adjuvant therapy was $67.6 \pm 8.0$ years (range, 55 to 85 years).

No statistically significant difference was found between the groups in average age at presentation, gender, final pathologic stage, or the number who received neoadjuvant radiation and chemotherapy (Table 1).

All 9 patients in the adjuvant therapy group were class I according to the Siewert classification scale. In the group that did not receive adjuvant therapy, 6 patients were Siewert class I, 4 were in class II, and 1 was in class III (Table 2 and Table 3).

Among the 9 patients who received adjuvant therapy for their positive gastric margin, 3 received radiation therapy alone directed at the remaining gastric margin, 4 had chemotherapy, and 2 had chemotherapy and radiation therapy. The survival time from the date of the operation, incidence of documented local tumor recurrence, development of distant metastatic disease, cause of death, and swallowing function at last follow-up for each of the patients in the adjuvant therapy group and no treatment group are summarized in Tables 2 and 3, respectively.

Table 4. Adjuvant Therapy Versus No Adjuvant Therapy for a Positive Margin After Transhiatal Esophagectomy-Summary of Outcomes

\begin{tabular}{lccc}
\hline & $\begin{array}{c}\text { Adjuvant } \\
\text { Therapy } \\
(\mathrm{n}=9)\end{array}$ & $\begin{array}{c}\text { No Adjuvant } \\
\text { Therapy } \\
(\mathrm{n}=11)\end{array}$ & $\begin{array}{c}p \\
\text { Value }\end{array}$ \\
\hline Survival (average days) & 477 & 455 & 0.898 \\
Local tumor recurrence (n) & 1 & 3 & 0.591 \\
Time to local tumor & 176 & 540 & 0.386 \\
$\quad$ recurrence (days) & & & \\
Swallowing Function & & 5 & 0.720 \\
$\quad$ Excellent & 5 & 0 & \\
$\quad$ Good & 1 & 2 & \\
Fair & 1 & 3 & \\
Poor & 2 & 1 & \\
$\quad$ Unknown & 0 & & \\
\hline
\end{tabular}




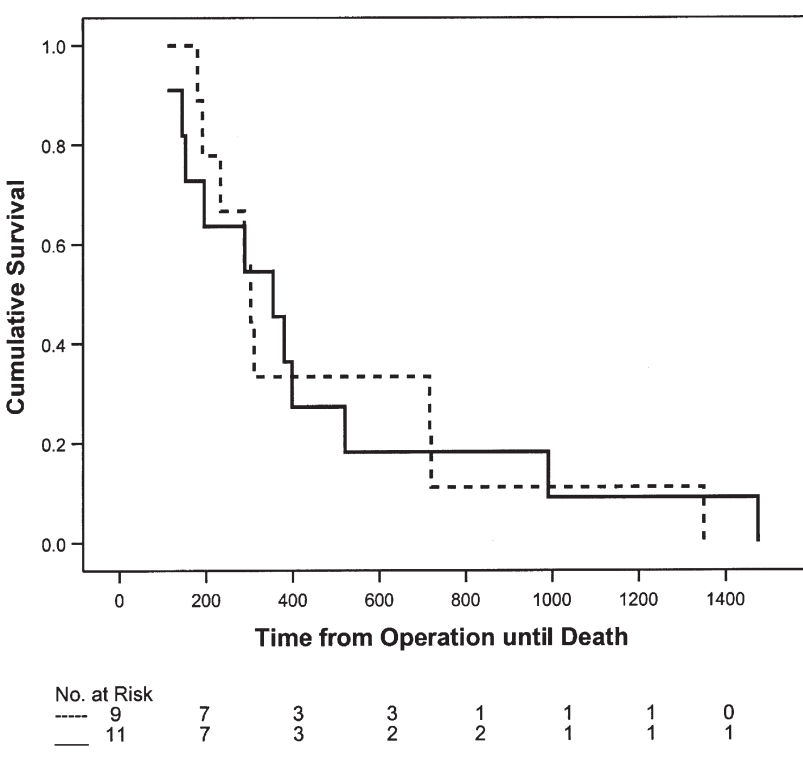

Fig 1. Kaplan-Meier analysis of survival with a positive gastric margin after transhiatal esophagectomy. Overall survival in number of days for patients receiving adjuvant therapy (dashed line) and for patients not receiving adjuvant therapy (solid line).

The average time from operation until death was 477 days in the group who received adjuvant therapy and 455 days in the group that did not receive adjuvant therapy (Table 4). The difference in survival between the two groups was not statistically significant $(p=0.898)$. The Kaplan-Meier survival functions for each group are shown in Figure 1.

Three patients $(27 \%)$ in the group that did not receive adjuvant therapy had a local tumor recurrence at the cervical esophagogastric anastomosis. This was discovered at endoscopy and biopsy performed to evaluate refractory postoperative dysphagia. One patient $(11 \%)$ in the group that did receive adjuvant therapy had a local tumor recurrence in the distal intrathoracic stomach, not at the cervical esophagogastric anastomosis. There was no statistically significant difference in the incidence of local tumor recurrence between the two groups ( $p=$ 0.591). In addition, the difference in the amount of time from esophagectomy to local tumor recurrence between the two groups was not significant ( $p=0.386$; Table 4).

Among those who received adjuvant therapy, swallowing function was excellent in 5 patients, good in 1, fair in 1 , and poor in 2. Swallowing function in the group that did not receive adjuvant therapy was excellent in 5 patients, fair in 2, and poor in 3 . Swallowing function data were not available for one patient in this latter group. The difference in swallowing function between those who received adjuvant therapy and those who did not was not statistically significant ( $p=0.720$; Table 4 ).

\section{Comment}

The general goal of oncologic surgical procedures is the complete removal of the primary tumor with an adequate margin of resection. In the University of Michigan experience with THE, partial proximal gastrectomy, and a cervical esophagogastric anastomosis for carcinoma of the distal esophagus and cardia, a gross gastric margin of 4 to $6 \mathrm{~cm}$ beyond palpable tumor generally provides a margin that is free of cancer on the final histologic examination.

The propensity of esophageal cancer to spread intramurally through the submucosal lymphatics has long been appreciated, with tumors of the GEJ spreading both proximally up the esophagus and distally down into the stomach [7-12]. Historically, a 10-cm margin both proximal and distal to gross tumor was advocated to achieve a negative margin of resection and to prevent local recurrence [13]. In the past three decades, as THE has been widely used for tumors of the distal esophagus and cardia, the ability to achieve a negative distal margin dividing the stomach 4 to $5 \mathrm{~cm}$ from macroscopic tumor has been recognized $[3,9,11,12]$. Nevertheless, in our 30-year experience with THE, $26(2.5 \%)$ of our 1044 patients undergoing the operation for adenocarcinoma of the distal esophagus and cardia have had a positive microscopic gastric margin. In 20 patients, this positive gastric margin could have had an impact on survival and quality of life.

Frozen sections on the margins of the resected specimen are not routinely used at our institution. The distal gastric margin alone is typically 8 to $12 \mathrm{~cm}$ long and would need to be sampled along its entire length on both the anterior and posterior surfaces to declare the margins to be free of cancer, an undertaking that is not clinically practical. When the resected specimen is examined on the back table in the operating room, however, and there is concern for mucosal changes extending to within $3 \mathrm{~cm}$ or less of the distal margin, a frozen section on the margin is sent. If this returns as positive, then further gastric resection is performed to achieve a negative margin. In fact, this was the case in 2 patients originally considered for this study. The first distal margin of resection was positive on frozen section; however, an additional 2 to $3 \mathrm{~cm}$ of stomach was resected, and this resulted in negative margins. Enough stomach remained to use the stomach as an esophageal replacement. These patients were not included in this study.

In some cases, however, the need to resect additional stomach may mean that there is no longer enough stomach left to use as an esophageal replacement, and a colon or jejunal interposition must be done. This is a rare occurrence, however, and in almost all of our patients, a more extensive gastric resection is not required. Thus, we continue to perform a partial proximal gastrectomy with a THE and CEGA rather than a hemigastrectomy or total gastrectomy as standard procedure for adenocarcinoma of the distal esophagus or cardia.

The news of a positive gastric margin, generally returning 1 week after the operation when the final pathology report becomes available, is disconcerting. When this situation occurs, further treatment must be individualized. If the patient is relatively young (ie, age 50 to 60 ), relatively healthy, has no evidence of metastatic disease on preoperative evaluation, and does not have significant 
nodal disease on final pathology, then consideration must be given to obtaining a barium enema to assess the suitability of the colon as an esophageal replacement. If the colon is found to be suitable, after an appropriate bowel preparation, the patient should be returned to the operating room where the intrathoracic stomach is taken down, resected, and a colon interposition performed. This is a sizable undertaking in someone who is recovering from a THE and CEGA, but it is warranted if the goal of therapy is total eradication of tumor.

Unfortunately, this scenario is not feasible in most patients with a positive gastric margin. They are typically elderly or debilitated and have evidence of nodal spread (stage III disease) on the final pathologic evaluation. In this group, the risk of reoperation to resect the intrathoracic stomach and perform a colon interposition outweighs the expected benefits, as a surgical cure is unlikely. If the surgeon simply takes down the stomach, resects it, and performs a cervical esophagostomy, metastases will develop in most patients before the planned later colon interposition can be accomplished, and comfortable eating is never achieved.

Is postoperative adjuvant therapy of any value in these patients? Of our 20 patients with a positive gastric margin after THE included in this analysis, 9 received some form of adjuvant therapy: radiation therapy in 3, chemotherapy in 4 , and combined chemotherapy and radiation therapy in 2. The difference in time from operation until death between those who did and did not receive some form of adjuvant treatment was not statistically significant.

One of the goals of postoperative adjuvant therapy in this setting is to prevent local tumor recurrence at the cervical esophagogastric anastomosis and thereby potentially prevent recurrent dysphagia. We found no statistically significant difference in swallowing function between the patients who received adjuvant therapy and those who did not. Interestingly, the 3 patients who had a local recurrence but did not receive adjuvant therapy had a recurrence at the cervical esophagogastric anastomosis, and the one patient from among those who had adjuvant therapy in whom a local recurrence developed had a recurrence distal to the anastomosis in the intrathoracic stomach. These differences in the location of the recurrent tumor did not, however, translate into any differences in swallowing function.

A similar percentage of patients in both groups had an excellent outcome with no dysphagia after their THE. In addition, a poor outcome, with ongoing dysphagia requiring regular dilatations, occurred in a similar percentage of patients in both groups. These results suggest that little if any additional benefit is gained from the perspective of improved swallowing in administering adjuvant therapy to patients who have a positive gastric margin after a THE, proximal partial gastrectomy, and CEGA.

Comfortable swallowing is the goal of any esophageal resection and reconstruction procedure, and the number of these patients who had "poor" swallowing at last follow-up was disturbing. Experience has taught that a cervical esophagogastric anastomotic leak results in an anastomotic stricture in $50 \%$ of patients in whom this complication occurs, but in this group, only $1(5 \%)$ of the 20 patients experienced a postoperative anastomotic leak. We have a liberal policy of outpatient cervical esophagogastric anastomotic dilatations in any patient who complains of any degree of cervical dysphagia after a THE, and we initiate instruction in self-dilatation if the dysphagia persists after one or two dilatations in the office. We have graded swallowing as "poor" in any patient who was issued an esophageal dilator for home use, although the patient may have been swallowing a regular diet comfortably between self-dilatation treatments.

Perhaps a more important issue than local tumor recurrence in these patients is that of metastatic disease. Overall, histologically documented distant metastatic disease developed in $12(60 \%)$ of the 20 patients, 6 of whom were in the adjuvant therapy group. Another 5 patients $(25 \%)$ were presumed to have metastatic disease at the time of death, although it was not confirmed histologically. Two of these were in the adjuvant therapy group. In 8 patients, including 4 in the adjuvant therapy group, metastatic disease was documented as a cause of death. An additional 9 patients, including 4 in the adjuvant treatment group, had presumed metastasis as the cause of death. With an average length of survival of only 466 days for all 20 patients, it is likely that most of them had unrecognized metastatic disease at the time of surgery.

Because adjuvant radiation therapy is intended to treat local rather than metastatic disease, it is understandable that survival was not increased in the patients who received only adjuvant radiation therapy. Chemotherapy is given to treat micrometastases, however, and thus would be expected to improve survival and decrease dysphagia caused by a local tumor recurrence in the intrathoracic stomach. In this small group, treatment with chemotherapy was apparently not effective at improving these outcomes.

This study specifically addressed the management of patients who have a histologically positive gastric margin after a transhiatal esophagectomy, proximal partial gastrectomy, and a cervical esophagogastric anastomosis for adenocarcinoma of the distal esophagus and gastric cardia. We acknowledge that the very small sample size and heterogenous nature of our patients limits the ability to draw meaningful conclusions about the appropriate treatment of these patients with a positive gastric margin. With such a small sample size, there would have to be large differences between the groups for a statistically significant difference to be detected. Although equivalence tests would provide an answer to the question of whether there are practical significant differences between the groups, they were not performed because such tests require an even larger sample size than the traditional difference tests reported.

The fact that the sample size of this study is so small validates the position that a THE, proximal partial gastrectomy, and CEGA is indeed an adequate operation for cancer of the distal esophagus and cardia. A positive distal gastric margin is a rare occurrence, and in almost 
all patients who undergo this operation, complete local tumor removal is achieved. The rarity of a positive gastric margin in these patients supports the practice of dividing the stomach 4 to $6 \mathrm{~cm}$ distal to gross palpable tumor and not routinely examining a frozen section of the distal gastric margin at the time of operation. These data also suggest that more extensive gastric resection is not necessary in most patients with carcinoma of the distal esophagus and cardia.

The lack of statistically significant differences in survival, ability to swallow comfortably, or local tumor recurrence in the intrathoracic stomach between patients who received adjuvant therapy and those who did not suggests the relative futility of this treatment in those with a positive gastric margin. In those patients in whom microscopic gastric infiltration by tumor is present more than 4 to $6 \mathrm{~cm}$ beyond palpable tumor, a more aggressive systemic neoplasm may well be present. Given the potential morbidity of postoperative adjuvant therapy and the lack of demonstrable benefit, the use of adjuvant therapy in this situation is questionable. Perhaps instead, most patients with a positive gastric margin should be allowed to convalesce and enjoy eating and living for as long as possible. These patients should be monitored for obvious recurrent or metastatic disease, which if symptomatic can then be treated. In these patients, efforts should be focused on providing adequate palliative care and the best possible quality of remaining life.

We would like to thank Dr Henry Appelman of the University of Michigan Department of Pathology for his assistance in reviewing the histopathology of the patients included in this study. We would also like to thank Laura Klem of the University of Michigan Center for Statistical Consultation and Research for assistance in the statistical analysis of these data and Debora Kayner for administrative assistance with this project.

\section{References}

1. Rudiger Siewert J, Feith M, Werner M, Stein HJ. Adenocarcinoma of the esophagogastric junction: results of surgical therapy based on anatomical/topographic classification in 1,002 consecutive patients. Ann Surg 2000;232:353-61.

2. Tytgat GN, Bartelink H, Bernards R, et al. Cancer of the esophagus and gastric cardia: recent advances. Dis Esophagus 2004;17:10-26.

3. Orringer MB, Marshall B, Iannettoni MD. Transhiatal esophagectomy: clinical experience and refinements. Ann Surg 1999;230:392-403.

4. Rice TW, Adelstein DJ, Chidel MA, et al. Benefit of postoperative adjuvant chemoradiotherapy in locoregionally advanced esophageal carcinoma. J Thorac Cardiovasc Surg 2003;126:1590-6.

5. Xiao ZF, Yang ZY, Liang J, et al. Value of radiotherapy after radical surgery for esophageal carcinoma: a report of 495 patients. Ann Thorac Surg 2003;75:331-6.

6. Japanese Esophageal Oncology Group. A comparison of chemotherapy and radiotherapy as adjuvant treatment to surgery for esophageal carcinoma. Chest 1993;104:203-7.

7. Law S, Arcilla C, Chu K, Wong J. The significance of histologically infiltrated resection margin after esophagectomy for esophageal cancer. Am J Surg 1998;176:286-90.

8. Miller C. Carcinoma of the thoracic esophagus and cardia, a review of 405 cases. Br J Surg 1962;49:507-22.

9. Casson AG, Darnton SJ, Subramanian S, Hiller L. What is the optimal distal resection margin for esophageal carcinoma? Ann Thorac Surg 2000;69:205-9.

10. Liebermann-Meffert D. Anatomy, embryology, and histology. In: Pearson FG, Cooper JD, Deslauries J, et al, eds. Esophageal surgery. 2nd ed. Philadelphia, PA: Churchill Livingstone; 2002:16-7.

11. Finley RJ. Adenocarcinoma of the esophagus and esophagogastric junction. In: Pearson FG, Cooper JD, Deslauries J, et al, eds. Esophageal surgery. 2nd ed. Philadelphia, PA: Churchill Livingstone; 2002:731.

12. Finley RJ, Inculet RI. The results of esophagogastrectomy without thoracotomy for adenocarcinoma of the esophagogastric junction. Ann Surg 1989;210:535-42.

13. Skinner DB, Belsey RHR. Management of esophageal disease. Philadelphia, PA: W. B. Saunders; 1988:757.

\section{DISCUSSION}

DR SHANDA H. BLACKMON (Houston, TX): I'd like to congratulate you on an excellent presentation. Doctor Orringer, I have two questions for you. If you had a positive margin and $85 \%$ of those patients died of metastatic disease, distant metastatic disease, what would you do with the remaining $15 \%$ of people who weren't dying of distant metastatic disease, and under what circumstances would you go back and reresect those patients if they have proven that they have no metastatic disease?

DR ORRINGER: It's a difficult call. The vast majority of patients who undergo an esophagectomy for carcinoma are not youngsters and are not in good health, and many of them have undergone chemotherapy and radiation therapy, and the thought of walking into the room on day 9 or 10 or 8 , after they're ready to go home on day 7 after the barium swallow, and say, "You know, your margin has come back positive. We need to get a barium enema and prep your colon and then go back and perhaps do a colon interposition, take everything down or disconnect you and leave you with a spit fistula and the tube, and then come back maybe and see if we'll hook you up if you don't have recurrent cancer within the coming year," it's a very, very difficult call to make. If it's the 50-year-old nurse that I was called about who had had that happen elsewhere and was otherwise in great shape, a thin patient, I'd take that patient back to the operating room and do exactly what we just talked about, but I think from this experience, we have to say that in the majority of our patients who are elderly and have suffered the ravages of cancer, it's just too much to undertake, and I think you have to be realistic about what you're going to do with these people and not in most cases offer them a big operation. In the operating room, we always put the specimen on the back table and open it and look to see what the specimen looks like. We do not routinely get frozen sections unless we're concerned, and the thing we're most concerned about is patients who have signet ring cell carcinomas, either the esophagus or the stomach, because they tend to spread, infiltrate the tissues, and those are the people that if we see the mucosa doesn't look absolutely normal when we open the specimen on the back table with a gross $4 \mathrm{~cm}$ to $5 \mathrm{~cm}$ margin or $6 \mathrm{~cm}$ margin beyond the gastroesophageal junction, we'll get a frozen section then, but that, of course, has its limitations as well since you can't freeze the entire margin. If necessary, we take an additional $2 \mathrm{~cm}$ and tubularize that stomach even more, which we hate to do but which many 
people have advocated doing, so you can do it and get away with it. I think it's a very difficult call, and it's the reason that we looked at this patient population, just for this reason, because it is such a difficult issue to deal with, both for the surgeon who wants to help his patient, to do an oncologically good operation, and for a patient who is faced with that knowledge. So it's a tough call. Clinical judgment.

DR BLACKMON: My second question is, to adequately power the study, you would have needed twice the numbers. With these numbers, you would have to have a $50 \%$ difference in any of your outcomes to show a statistically significant difference. I wondered if you considered combining your patients with those of other institutions to adequately have enough power to show a difference.

DR ORRINGER: It's a point that's very well taken. It's a small series, and I think the fact that it's a small series justifies the oncologic relevance of the operation in the vast majority of people. If we can only uncover 20 patients in 1,400 or 1,500 patients with cancers who have been operated upon in all but $2 \%$, we've done a pretty good job. But I agree that a multiinstitutional study would be of value, provided that all the difficulties of multi-institutional studies are dealt with-the same operation, the same type of preoperative staging, the same type of preoperative chemotherapy, and so forth. It's needed, and hopefully our new international effort that is under way may bear fruit if we can standardize this treatment. And the Cleveland Clinic group is trying to organize just such an endeavor.

DR MARK J. KRASNA (Baltimore, MD): I'm a little nervous, Mark. I think this is the first time I'm ever going to disagree with you in public. Having said that, I think it's an excellent presentation. I'm perplexed. I have helped author two large series of open transthoracic esophagectomies-the paper with Dr Ellis with more than 450 patients and our most recent experience, also with a combined number of more than 400 patients. I have to admit I've never seen a positive gastric margin in either of those series. I've seen a few positive proximal margins. I've seen some positive residual Barrett's. And I think what Dr Blackmon mentioned, and actually what you have alluded to, is that if in fact there is a positive distal margin, that is because you are doing such a better job than I am at making your gastric tube, you're preserving "too much" stomach. I have actually over the last decade gone to making relatively narrow, 4-cm-wide tubes. It may be because I make a much narrower tube, and probably I have more leaks therefore than you do, that I'm never seeing these positive margins because I'm getting them in the specimen. So I do want you to comment on that.

My other question, why not, therefore, do frozens on everybody? I mean you're doing such a great job with $98 \%$, and this is clearly residual tumor that you're leaving behind, you have excellent survival otherwise, so why not do the frozen if that's all it's going to take, because you're going to eradicate this problem.

DR ORRINGER: Mark, do you do a frozen?

DR KRASNA: I don't, but I've never seen a positive distal margin.
DR ORRINGER: Well, I've only seen it in $2 \%$ of my patients, so that's why I don't do it. I can't argue with you. I think to argue that if we had a wider margin, we wouldn't have the $2 \%$ that we have, I can't argue. You know that we have tried to preserve stomach and to maximize vascularity of the specimen, but when you're doing an oncologic operation, you're weighing your desire to maximize vascularity of the conduit that you're using versus trying to do a better cancer operation. So, as I've said, when we look at that tube, if the mucosa looks perfectly normal, I don't know what to tell them to do a frozen section on. If I see gross abnormality or I'm concerned, I'll put a suture on it and say, "Freeze this. This is the area that I'm most concerned about," but they can't freeze the entire circumference of that margin, and I think we just have to use, as I said, clinical judgment. But your point is well taken. And, of course, that's what we do. When we're worried in the operating room, we make a wider gastric tube. We don't do it very often, but we do exactly that.

DR KRASNA: And I have just one follow-up detail. On most of the clinical trials that I've done, both at Maryland and the national trials, the cutoff for allowance to enroll a patient in the neoadjuvant studies was within $2 \mathrm{~cm}$ of the bottom of the gastroesophageal junction. At your institution, is it your institutional experience to allow more distal spread and still include them in your esophageal neoadjuvant, or do you also do it roughly $2 \mathrm{~cm}$ or $3 \mathrm{~cm}$ ? I'm wondering if these were bigger, bulkier, distal tumors.

DR ORRINGER: No. We follow the same tenets that you do.

DR JOE B. PUTNAM (Nashville, TN): I have two short questions. First, do you remember the percent of patients with signet ring carcinoma in the positive gastric margin group?

DR ORRINGER: It's not large, but it's real. There were maybe two or three of them.

DR PUTNAM: There seems to be an association with signet ring.

DR ORRINGER: It's more common. It wasn't a huge volume, but several of them, two or three, were signet rings.

DR PUTNAM: And the patients who died of metastatic disease, did the patients have dysphagia or did they have normal oral alimentation until they died?

DR ORRINGER: That's the point, that most of them ate well but died of distant metastatic disease. So that influences our recommendation to hammer them hard before they have symptoms after this.

DR PUTNAM: Thank you.

DR ORRINGER: Let me just tell you that Paul was also an M3 student when he began this, and he's now in his sixth month of general surgery residency. So another fine job for a medical student project and guys that we're trying to recruit into our specialty. 